\title{
Highly Concentrated, Conductive, Defect-free Graphene Ink for Screen-Printed Sensor Application
}

Cite as

Nano-Micro Lett.

(2021) 13:87

Received: 16 November 2020

Accepted: 29 January 2021

Published online: 8 March 2021

(C) The Author(s) 2021

\author{
Dong Seok Kim ${ }^{1}$, Jae-Min Jeong ${ }^{2}$, Hong Jun Park ${ }^{1}$, Yeong Kyun Kim ${ }^{1}$, \\ Kyoung G. Lee ${ }^{3 凶}$, Bong Gill Choi ${ }^{1 凶}$
}

\section{HIGHLIGHTS}

- Ultrathin and defect-free graphene ink is prepared through a high-throughput fluid dynamics process, resulting in a high exfoliation yield (53.5\%) and a high concentration $\left(47.5 \mathrm{mg} \mathrm{mL}^{-1}\right)$.

- A screen-printed graphene conductor exhibits a high electrical conductivity of $1.49 \times 10^{4} \mathrm{~S} \mathrm{~m}^{-1}$ and good mechanical flexibility.

- An electrochemical sodium ion sensor based on graphene ink exhibits an excellent potentiometric sensing performance in a mechanically bent state.

- Real-time monitoring of sodium ion concentration in sweat is demonstrated.

ABSTRACT Conductive inks based on graphene materials have received significant attention for the fabrication of a wide range of printed and flexible devices. However, the application of graphene fillers is limited by their restricted mass production and the low concentration of their suspensions. In this study, a highly concentrated and conductive ink based on defect-free graphene was developed by a scalable fluid dynamics process. A high shear exfoliation and mixing process enabled the production of graphene at a high concentration of $47.5 \mathrm{mg} \mathrm{mL}^{-1}$ for graphene ink. The screen-printed graphene conductor exhibits a high electrical conductivity of $1.49 \times 10^{4} \mathrm{~S} \mathrm{~m}^{-1}$ and maintains high conductivity under mechanical bending, compressing, and fatigue tests. Based on the as-prepared graphene ink, a printed electrochemical sodium ion $\left(\mathrm{Na}^{+}\right)$sensor that shows high potentiometric sensing performance was

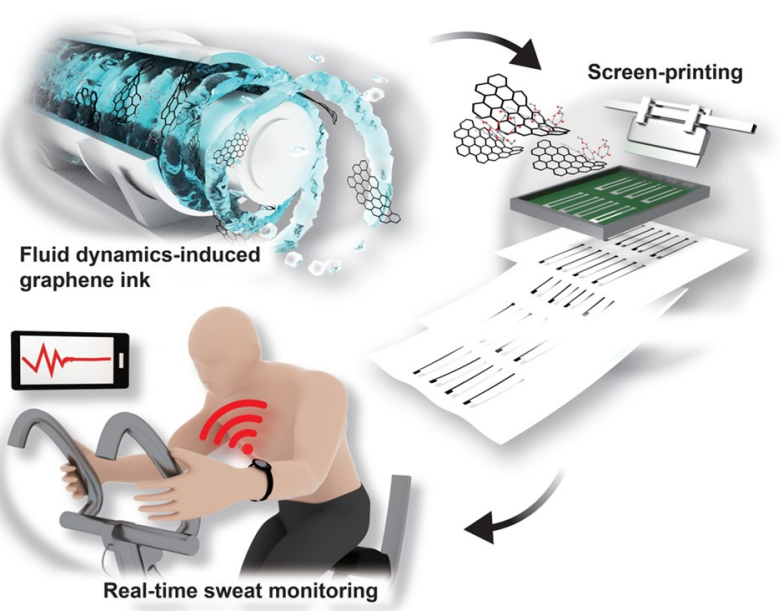
fabricated. Further, by integrating a wireless electronic module, a prototype $\mathrm{Na}^{+}$-sensing watch is demonstrated for the real-time monitoring of the sodium ion concentration in human sweat during the indoor exercise of a volunteer. The scalable and efficient procedure for the preparation of graphene ink presented in this work is very promising for the low-cost, reproducible, and large-scale printing of flexible and wearable electronic devices.

KEYWORDS Graphene ink; Fluid dynamics; Screen printing; Ion sensor; Real-time monitoring

Dong Seok Kim, Jae-Min Jeong, and Hong Jun Park have contributed equally to this work.

$\triangle$ Kyoung G. Lee, kglee@nnfc.re.kr; Bong Gill Choi, bgchoi@kangwon.ac.kr

1 Department of Chemical Engineering, Kangwon National University, Samcheok, Gangwon-do 25913, Republic of Korea

2 Resources Utilization Research Center, Korea Institute of Geoscience and Mineral Resources, Daejeon 34132, Republic of Korea

3 Center for Nano Bio Development, National Nanofab Center, Daejeon 34141, Republic of Korea 


\section{Introduction}

Wearable chemical sensor technology has rapidly developed in recent years with applications in personalized healthcare, fitness management, and medical diagnostics because it provides continuous and real-time physiological information with regard to health status changes to the wearer [1-5]. A wearable wireless sensor device typically contains the following key functional units: a substrate, active materials, and an integrated circuit module [6,7]. The active materials, composed of sensing and electrical components, are printed or transferred to flexible or stretchable substrates such as flexible plastics, paper, textiles, and soft polymer films [8, 9]. To further provide flexibility or stretchability to sensor devices, mechanically structured design approaches have been used in the manufacture of wearable devices, including wavy, fractal, origami, kirigami, and island-interconnected structures [10-14]. The as-obtained sensing platforms selectively recognize human body fluids such as saliva, tear, sweat, and urine as biochemical markers, and the collected data can be transmitted to the user's smartphone application $[15,16]$. However, saliva, tear, and urine-based sensors are difficult for continuous and noninvasive health monitoring in a wearable platform because of limited location and sample conditions [17-20]. In contrast, sweat is easy to access noninvasively through the skin and is rich in important analytes, such as electrolytes (e.g., $\mathrm{Na}^{+}, \mathrm{Cl}^{-}, \mathrm{K}^{+}$, and $\mathrm{pH}$ ) and metabolites (e.g., glucose, lactate, and cortisol) [21, 22]. Recent studies have shown that potentiometric ion sensors in a wearable format are useful for real-time monitoring of dynamic changes in ion concentrations in sweat during an individual's activities [23, 24]. The on-body monitoring of sodium and chloride ions in sweat can be utilized to indicate dehydration status and to diagnose cystic fibrosis $[25,26]$. The potassium level in sweat is related to muscle activity [27], and $\mathrm{pH}$ monitoring can be used for the management of chronic wounds [28]. A number of wearable sweat sensors have recently been developed in many different device forms, such as multi-sensor arrays [29], textile-based potentiometric sensors [30], smart bandages [31], patches [32], and tattoos [33], and these sensors are capable of monitoring electrolytes, metabolites, heavy metals, and toxic gases in human body fluids [34-36]. Despite the development of state-of-the-art sensors, considering their many applications in point-of-care diagnostics and exercise monitoring, the wearable sensor market requires that the sensor platforms are low cost, disposable, and amenable to mass production with high reproducibility.

Screen printing is a high-speed, cost-effective, reproducible, and large-scale process for the fabrication of sensor electrodes or devices by the transfer of inks onto flexible substrates through a stencil mask [37, 38]. The properties of functional inks, such as their conductivity, concentration, viscosity, and flexibility, are key factors for realizing highperformance wearable chemical sensors [39, 40]. Graphene, a two-dimensional carbon nanosheet, has gained considerable attention as a promising ink material for the construction of next-generation printed flexible electronics and devices because of its outstanding mechanical, thermal, chemical, and electrical properties [40-42]. Graphene materials are typically prepared from graphite by chemical oxidative exfoliation and liquid-phase exfoliation (LPE) methods [43, 44]. The oxidation of graphite and subsequent exfoliation results in a large quantity of graphene oxide (GO) nanosheets dispersed in polar solvents such as water and alcohols [43]. An insulating GO can be converted into conductive reduced graphene oxide (RGO) by chemical or annealing reduction [45]. However, the unrepaired defects and distorted structure of RGO lead to low electrical conductivity, thus limiting its application in conductive inks. In this regard, there has been much research focused on the direct delamination of graphite by the LPE method to potentially obtain suspensions of defect-free graphene without any chemical functionalization [46-48]. The resultant graphene is used in the form of a highly conductive ink, paste, or composite for printing many devices [49, 50]. However, LPE still provides a low yield $(<12 \%)$ or graphene concentration $\left(<10 \mathrm{mg} \mathrm{mL}^{-1}\right)$, which is a significant limitation in the preparation of exfoliated graphene [51, 52]. Although a low-power ultrasonication resulted in a high yield of $33 \%$ for preparation of defectfree single-layer graphene for inkjet-printed graphene electronics, a long reaction time of $9 \mathrm{~h}$ and a low concentration of $\sim 0.11 \mathrm{~g} \mathrm{~L}^{-1}$ limit this to large-scale screen-printing applications [53]. The use of an ultrasonic bath or probe in LPE is time-consuming because of its energy is applied locally and non-homogeneously $[54,55]$, and thus, the exfoliation process is not suitable for industrial scale-up. Moreover, the concentration of graphene needs to be increased in order to develop screen-printing inks through post-processing procedures such as repetitive centrifugation and redispersion, which increase the preparation time. To address these issues, the fluid dynamics process has emerged as a rapid 
and scalable method to produce defect-free and large-lateral size graphene sheets from direct exfoliation of graphite using suitable solvents and water $[51,56]$. Typically, graphite flakes are delaminated by fluid dynamics-induced shear exfoliation. Other factors such as pressure, cavitation, and collision and efficient mixing behavior contribute to the exfoliation process. To date, various fluid dynamics-based exfoliation approaches have been attempted for graphite exfoliation, including vortex fluidic flow, pressure-driven fluid dynamics, Taylor-Couette (TC) flow, and mixer-driven fluid dynamics. In particular, a TC flow generated in the small gap between two rotating cylinders provides high wall shear stress and pressure and efficient mass transfer, and thus, these fluidic features can efficiently produce defect-free and few-layer graphene sheets in a large quantity.

Here, we report the scalable and efficient production of highly concentrated and conductive inks of defect-free graphene by a TC flow-induced exfoliation and mixing process. The delamination of graphite resulted in a high yield of exfoliated graphene (ex-Gr) of $53.5 \%$, which was obtained at a high suspension concentration of $47.5 \mathrm{mg} \mathrm{mL}^{-1}$. The screenprinted ex-Gr conductor exhibited a high electrical conductivity of $1.49 \times 10^{4} \mathrm{~S} \mathrm{~m}^{-1}$ and good mechanical flexibility. Moreover, we demonstrated that the printed ex-Gr conductor can be used as an electrochemical ion-sensing electrode for the potentiometric detection of sodium ions $\left(\mathrm{Na}^{+}\right)$. The resulting $\mathrm{Na}^{+}$sensor exhibited high sensitivity, fast response time, good repeatability, and good selectivity. As a proofof-concept, we performed on-body testing on a volunteer wearing the wireless electronically integrated $\mathrm{Na}^{+}$-sensing device during a stationary bike exercise to demonstrate the real-time monitoring of $\mathrm{Na}^{+}$concentration in his sweat.

\section{Experimental}

\subsection{Materials}

Sodium ionophore $X$, sodium tetrakis[3,5bis(trifluoromethyl)phenyl]borate (Na-BARF), bis(2-ethylhexyl) sebacate (DOS), poly(vinyl chloride) (PVC) with high molecular weight, sodium chloride, potassium chloride, magnesium chloride, calcium chloride, ammonium chloride, ethyl cellulose with viscosity $10 \mathrm{cP}$, terpineol, BUTVAR® B-98 (polyvinyl butyral, PVB), methanol, and tetrahydrofuran (THF) were purchased from Sigma Aldrich. Ethyl alcohol was purchased from Samchun Chemicals Co. Ltd. $\mathrm{Ag}$ and $\mathrm{Ag} / \mathrm{AgCl}$ inks were obtained from Asahi chemical research laboratory Co. Ltd. Deionized (DI) water was prepared by using a Milli-Q unit (resistivity: $18.2 \mathrm{M} \Omega \mathrm{cm}^{-1}$, Millipore System, Darmstadt, Germany).

\subsection{Preparation of ex-Gr Inks}

The conductive and defect-free ex-Gr inks were prepared by the fluid dynamics-assisted exfoliation and mixing process. A mixture of graphite and EC (9:1 in weight) dispersed in terpineol/ethanol (5:5 in volume) was loaded in a reactor and processed at $2000 \mathrm{rpm}$ for $2 \mathrm{~h}$. The fluidic reactor is made by combining two inner-rotational and outer-stationary cylinders based on stainless steel. At a specific rotation speed of $2000 \mathrm{rpm}$, the reactor generates the Taylor vortexes entire reactor volume that provides unique mass transfer and shear field [57]. Different concentrations of graphite $(1,5,10,50$, 100 , and $200 \mathrm{mg} \mathrm{mL}^{-1}$ ) were tested. After the exfoliating and mixing process, the exfoliated dispersion was collected by centrifugation at $250 \mathrm{~g}$ for $30 \mathrm{~min}$. And then, the ultrasmall size of graphene was also removed by centrifugation at $3200 \mathrm{~g}$ for $60 \mathrm{~min}$. The as-obtained ex-Gr inks were heated at $225{ }^{\circ} \mathrm{C}$ for $24 \mathrm{~h}$ under vacuum to remove terpineol and ethyl cellulose, and then weighed by using an electronic scale XSR105 (Mettler toledo) to evaluate the exfoliation yield and final concentration of ex-Gr nanosheets.

\subsection{Preparation of $\mathrm{Na}^{+}$Sensor Based on Screen-Printed ex-Gr Electrode}

Prior to fabricating the $\mathrm{Na}^{+}$sensors, the electrical circuit electrodes were prepared using a screen printer SJ-7450S (Sung-Jin tech Ltd., Korea) and a custom stainless-steel mask designed with two-electrode patterns. The ex-Gr and $\mathrm{Ag}$ inks were printed onto a flexible substrate of polyethylene terephthalate (PET) film, in which ex-Gr and Ag inks were used as electrical circuits for the working and reference electrodes, respectively. The resultant electrode was annealed at $200{ }^{\circ} \mathrm{C}$ for $120 \mathrm{~min}$. Custom stainless-steel masks were made with different thicknesses of $25-45 \mu \mathrm{m}$, corresponding to the thickness of the printed ex-Gr conductors in $20-41 \mu \mathrm{m}$. The working electrodes were prepared by coating the $\mathrm{Na}^{+}$-ISM onto the surface of printed ex-Gr. The $\mathrm{Na}^{+}$-ISM is consisted of Na ionophore $\mathrm{X}(1 \%$ weight 
by weight, w/w), Na-BARF (0.5\%, w/w), PVC (31.5\%, $\mathrm{w} / \mathrm{w})$, and DOS $(67 \%, \mathrm{w} / \mathrm{w})$ in THF $(1 \mathrm{~mL})$. For a reference electrode, $\mathrm{Ag} / \mathrm{AgCl}$ ink was coated onto the reaction part of the printed Ag-electrical circuit. To ensure electrochemical stability of the reference electrode, the as-obtained $\mathrm{Ag}$ / $\mathrm{AgCl}$-coated electrode was coated by a casting method using a mixture $50 \mathrm{mg}$ of $\mathrm{NaCl}$ and $78 \mathrm{mg}$ of PVB dissolved in $1 \mathrm{~mL}$ methanol solution $[5,58]$. The resultant $\mathrm{Na}^{+}$sensors were dried at room temperature for $24 \mathrm{~h}$.

\subsection{Characterization and Measurements}

Field-emission scanning electron microscope (FE-SEM, Hitachi S-4800) and field-emission transmission electron microscope (FE-TEM, JEOL Ltd. JEM-2100F HR) were performed to investigate the morphology and microstructure of ex-Gr nanosheets and printed ex-Gr electrodes. The atomic force microscopy (AFM) images were obtained by an AFM-Raman spectrometer (INNOVA-LABRAM HR800, Bruker Co., Ltd.). High-resolution X-ray diffraction (XRD) was performed on a D/MAX-2500 V with a $\theta / \theta$ goniometer equipped with a $\mathrm{Cu} \mathrm{K} \alpha$ radiation generator. X-ray photoelectron spectroscopy (XPS, Thermo MultiLab 20,000) and Raman microscopy (ARAMIS, Horiba Jobin Yvon) were performed to investigate chemical structure of graphite and ex-Gr. The electrical conductivity of printed ex-Gr conductors was measured using four-point probe system (Conductivity meter, Loresta-GX MCP-T700). The ex-Gr inks were screen-printed onto various substrates of PET, polyimide (PI), and paper, followed by annealing to different temperature $\left(100,125,200\right.$, and $\left.225^{\circ} \mathrm{C}\right)$. For measure electrical conductivity, the as-obtained ex-Gr conductors were cut to $1 \mathrm{~cm} \times 1 \mathrm{~cm}$ size. The electrochemical performances of $\mathrm{Na}^{+}$sensors were evaluated using an electrochemical instrument of CHI 760 E (CH Instruments, USA). The different concentrations of $\mathrm{NaCl}$ solution $\left(10^{-1}-10^{-4} \mathrm{M}\right)$ were prepared by diluting $1 \mathrm{M} \mathrm{NaCl}$ solution. Fatigue test and bending test were performed using digital multimeters (Protek HC-81). Bending and compressing tests were measured resistance of bending angles while reduced length using ex-Gr screen-printed electrodes of $10 \times 1 \mathrm{~cm}^{2}$ size. Fatigue test was measured resistance while bending over 1400 cycles. Cyclic voltammetric curves of screen-printed graphite and ex-Gr electrodes were measured at a scan rate of $50 \mathrm{mV} \mathrm{s}^{-1}$ in $10^{-1} \mathrm{M} \mathrm{NaCl}$. Sensitivity of bending and normal state was measured different concentration of $\mathrm{NaCl}$ solutions $\left(10^{-1}-10^{-4} \mathrm{M}\right)$. Long-term stability of sodium sensor was measured for $15 \mathrm{~h}$ in $10^{-1} \mathrm{M} \mathrm{NaCl}$ solution. Ion selectivity of sodium sensor was evaluated using various ion solutions that is $10-20 \mathrm{mM} \mathrm{NaCl}, 5 \mathrm{mM} \mathrm{KCl}, 0.5 \mathrm{mM} \mathrm{NH}_{4} \mathrm{Cl}$, $0.5 \mathrm{mM} \mathrm{MgCl}_{2}$, and $0.5 \mathrm{mM} \mathrm{CaCl}_{2}$. The repeatability test of the $\mathrm{Na}^{+}$sensor was performed using a titrated cycle of $10^{-1}$ to $10^{-2}$ to $10^{-3}$ to $10^{-4} \mathrm{M}$, in which the sensors were used continuously without washing steps.

\subsection{On-body Sweat Measurements}

A wristwatch-type wearable device was fabricated by integrating $\mathrm{Na}^{+}$sensor and a wireless electronic printed circuit board (PCB) module into 3D printed wristwatch. The PCB system was fabricated according to our previous reports $[58,59]$. A block diagram of PCB system is described in Fig. S1. The PCB consists of the electrical units and communication modules, involving electrochemical ion sensor, interface circuits, a microcontroller unit (64 MHz, Arm Cortex-M4), a regulator (LP5907-3.3), an analog to digital converter (ADC, ADS 1114), and Bluetooth low energy (BLE). A lithium-ion battery $(3.7 \mathrm{~V}$ ) was used as the power source. The $\mathrm{Na}^{+}$sensor was connected with PCB module using an electrical jumper wire (male to female). The Kapton tape for the insulation of $\mathrm{Na}^{+}$sensor was attached at both end sides of the electrodes and PCB module. The PCB collects continuously the $\mathrm{Na}^{+}$ concentration data in sweat and transfer to a smartphone via Bluetooth. The real-time monitored $\mathrm{Na}^{+}$concentration was displayed in a mobile application. On-body tests were according to compliance with relevant guidelines approved by the institutional review board of Kangwon National University (KWNUIRB-2018-12-002-001). A healthy volunteer (male aged 28-years-old) provided written informed consent after understanding the on-body test guidelines. A commercial heart rate sensor device was used together. Prior to on-body sweat test, a volunteer's wrist was cleaned with soap and water and then dried using alcohol. Real-time monitoring of sweat sensors was performed by exercising on a stationary bike for $20 \mathrm{~min}$ at room temperature. 


\section{Results and Discussion}

\subsection{Preparation and Characterization of ex-Gr Inks}

Figure 1a shows the simple and efficient preparation process for conductive ex-Gr inks. As an exfoliation method, a fluid dynamics-induced delamination process was employed to obtain a high yield of defect-free ex-Gr at a high concentration in the suspension. The use of the TC flow allowed uniform and high shear rates and fast mass transfer, resulting in high-throughput production of ex-Gr dispersed in suitable solvents [60]. Typically, a mixture of graphite and EC (9:1 in weight) dispersed in terpineol/ethanol (5:5 in volume) was loaded in a fluid dynamic reactor and processed for $2 \mathrm{~h}$. The process was investigated with different concentrations of graphite $\left(1,5,10,50,100\right.$, and $\left.200 \mathrm{mg} \mathrm{mL}^{-1}\right)$. EC was used as a stabilizer to efficiently exfoliate and suspend graphene flakes in an organic solvent such as a mixture of terpineol and ethanol. EC also served as a binder; it enhanced adhesion between graphene and printing substrates. After centrifugation to remove unexfoliated graphite, a large quantity of high-quality ex-Gr was obtained. The diverse fluid dynamics forces, including shear, pressure, cavitation, and collision forces [57], enable the efficient and rapid exfoliation of graphite, resulting in a high yield and high concentration of ex-Gr suspension. The yield and concentration of ex-Gr depended on the initial graphite concentration. The maximum yield of the as-obtained ex-Gr suspension of $53.5 \%$ was obtained at a low initial graphite concentration of $1 \mathrm{mg} \mathrm{mL}^{-1}$, and the concentration reached as high as $47.5 \mathrm{mg} \mathrm{mL}^{-1}$ when the initial graphite concentration was $200 \mathrm{mg} \mathrm{mL}^{-1}$ (Fig. 1b, c). A highly viscous and concentrated graphite loading over $200 \mathrm{mg} \mathrm{mL}^{-1}$ did not allow us to provide a TC-induced exfoliation process because a high viscous flow limits the rotating cylinders to a small distance between the two cylinders. Remarkably, the use of a fluid dynamics reactor enabled us to obtain highly conductive and concentrated graphene suspension at a large-scale and at a fast speed. A high exfoliation yield of 53.5\% and a high concentration of $47.5 \mathrm{mg} \mathrm{mL}^{-1}$ of this process are notably better than that of conventional methods such as sonication, ballmilling, shearing, and processing with a mortar (Table S1, Supporting Information) [51, 60]. TEM images reveal highly exfoliated nanosheets and high-quality crystalline structure of ex-Gr (Fig. 1d). The flake size of ex-Gr was statistically analyzed from SEM images (Fig. 1e). For SEM observation, (a)

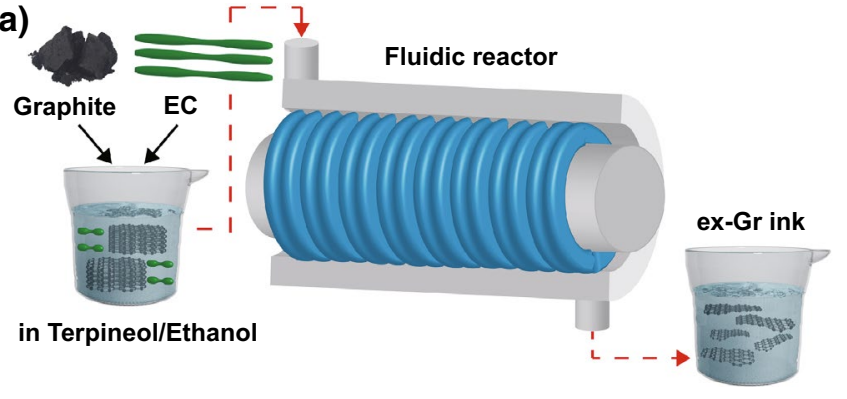

(c)

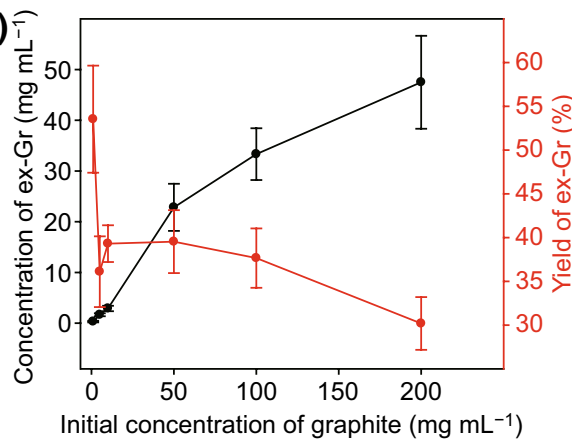

(d) (b)

Low con. ex-Grinks High con.
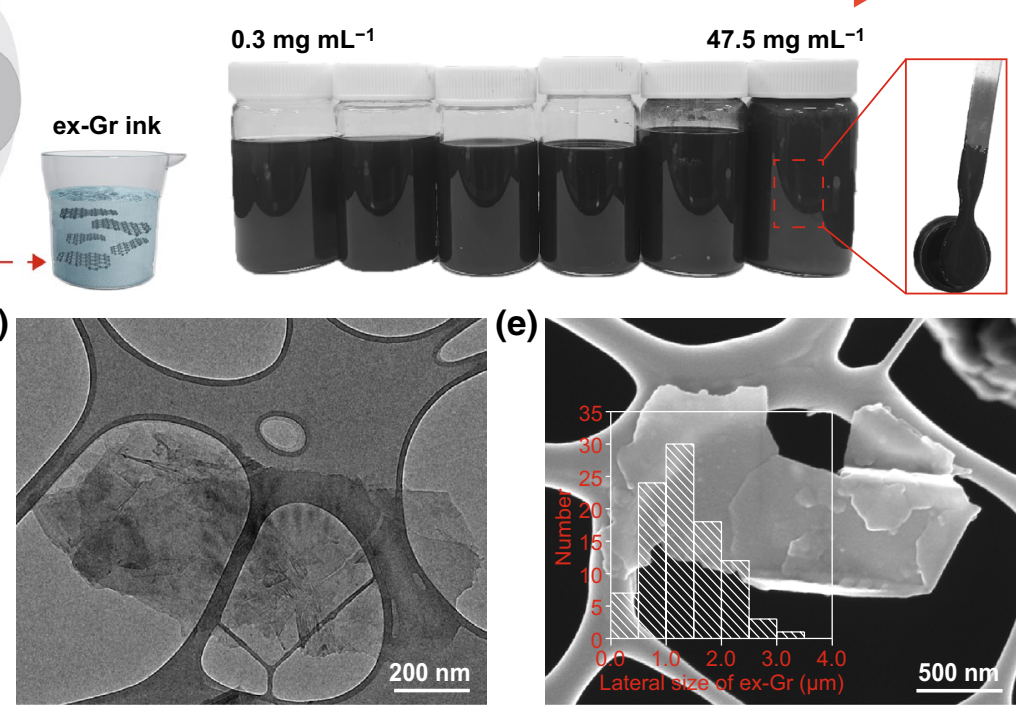

Fig. 1 a Schematic illustration of the fluid dynamics process used for the preparation of ex-Gr ink. b Photograph image of the ex-Gr inks at different concentrations $\left(0.3-47.5 \mathrm{mg} \mathrm{mL}^{-1}\right)$. c Concentrations and yields of the ex-Gr obtained at different initial concentrations of the graphite $\left(1-200 \mathrm{mg} \mathrm{mL}^{-1}\right)$. d TEM and e SEM images of the ex-Gr sheet (Inset: histogram for the lateral size distribution of ex-Gr) 
the ex-Gr suspension was drop-casted onto a TEM grid, and was then washed and annealed to remove the solvents and surfactant. According to the analysis of more than 90 sheets in the SEM images, the lateral size distribution of ex-Gr is in the range $0.29-3.25 \mu \mathrm{m}$ and its average lateral size is $1.10 \pm 0.84 \mu \mathrm{m}$. The thickness of ex-Gr was determined to be $1.2 \pm 0.8 \mathrm{~nm}$ via AFM measurement (Fig. S2). Based on this result, the as-obtained ex-Gr consists of few-layer nanosheets less than five layers, indicating multilayered graphene. The lateral size and thickness of few-layered ex-Gr obtained from the TC flow-induced exfoliation process were similar to previously reported values obtained from shearing and microfluidic exfoliation processes [61-65].

The crystallinity and chemical quality of ex-Gr was investigated by Raman spectroscopy, XPS, and XRD. The Raman spectrum of ex-Gr has three characteristic peaks at 1357 , 1582 , and $2725 \mathrm{~cm}^{-1}$, corresponding to the $\mathrm{D}, \mathrm{G}$, and $2 \mathrm{D}$ bands (Fig. 2a) [66]. The analysis of the Raman D/G band intensity ratio $\left(I_{\mathrm{D}} / I_{\mathrm{G}}\right)$ allows us to obtain information on the extent of structural disorder or defects in ex-Gr sheets [67]. The value of $I_{\mathrm{D}} / I_{\mathrm{G}}$ was estimated to be 0.21 , which is much lower than the previously reported values of $0.3-2.5$ for graphene materials obtained by the sonication-based LPE methods [54]. This result indicates the absence of basalplane defects in the ex-Gr sheets. Compared to the 2D peak of graphite, ex-Gr exhibited a more symmetrical and broader 2D peak; that is, the full width at half maximum increased from $98 \mathrm{~cm}^{-1}$ (graphite) to $115.5 \mathrm{~cm}^{-1}$ (ex-Gr). This result indicates that the ex-Gr sheets predominantly have a monoor few-layer structure [68]. The high-resolution C 1s XPS profile (Fig. 2b) of ex-Gr shows a sharp and prominent peak at $284.18 \mathrm{eV}$ corresponding to the $\mathrm{C}-\mathrm{C}$ bond of graphite [67]. After deconvolution, the C 1s peak showed no peaks of oxygenated carbon (e.g., $\mathrm{C}-\mathrm{O}$ and $\mathrm{C}=\mathrm{O}$ ) [67]. Compared to XRD pattern of graphite, ex-Gr exhibited significantly decreased diffraction peak, indicating the presence of abundant exfoliated graphene sheets (Fig. 2c) [60]. According to these results, the fluid dynamics process yields ultrathin exfoliated graphene sheets with a high-quality graphitic structure.

\subsection{Electrical and Mechanical Characterization of Screen-printed ex-Gr Conductor}

The highly concentrated and conductive ex-Gr dispersed in terpineol/ethanol solution could be readily used as a screen-printable conductive ink. Based on comparison of the TC flow-induced ink preparation and other conventional methods, the TC flow-induced process is faster and more efficient for obtaining a high concentration of ex-Gr inks (Table S1). It should also be noted that the ultrahigh concentration ( $>47.5 \mathrm{mg} \mathrm{mL}^{-1}$ ) of the ex-Gr ink allowed us to eliminate the post-evaporation step and the addition of binders often required for the screen-printing process. Figure $3 \mathrm{a}$ shows screen-printed ex-Gr lines with widths of 420, 330, and $200 \mu \mathrm{m}$. The plane-view SEM image of the printed lines (Fig. 3b) displays highly compact and interconnected networks of ex-Gr flakes, which is favorable for high electron transfer. The cross-sectional SEM image in Fig. 3c shows a good adhesion at the interface of the printed ex-Gr and PET substrate. A small inner volume is observed between the ex-Gr layers (Inset of Fig. 3c). To minimize the influence of the inner volume on the electrical and mechanical properties of the printed ex-Gr conductor, the annealing process was performed on a screen-printed ex-Gr conductor. The conductivity of the printed ex-Gr conductor was significantly
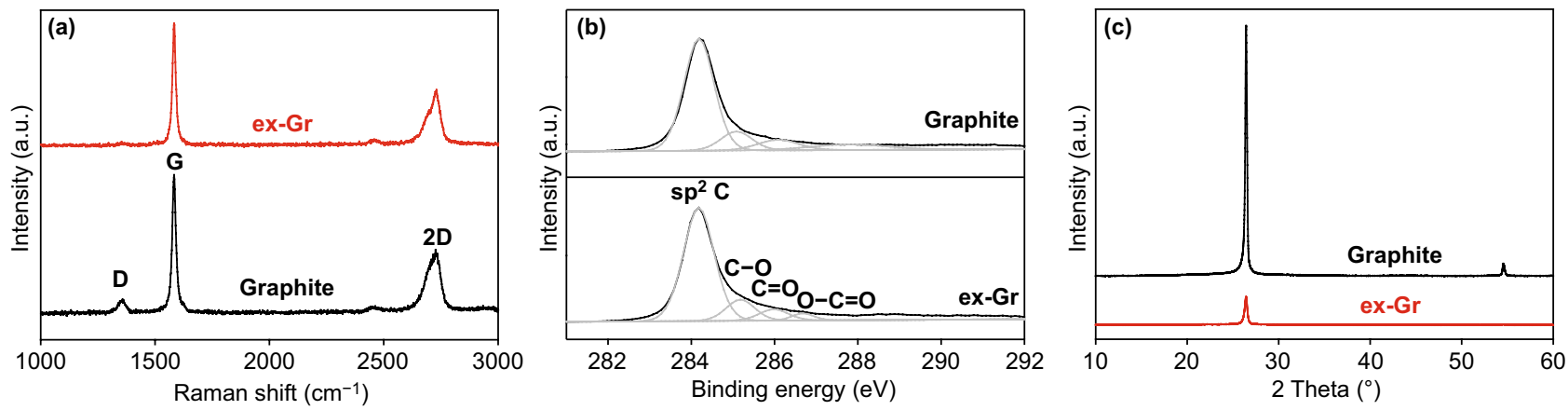

Fig. 2 a Raman spectra, b XPS C 1 s spectra, and $\mathbf{c}$ XRD patterns of graphite and ex-Gr 
(a)

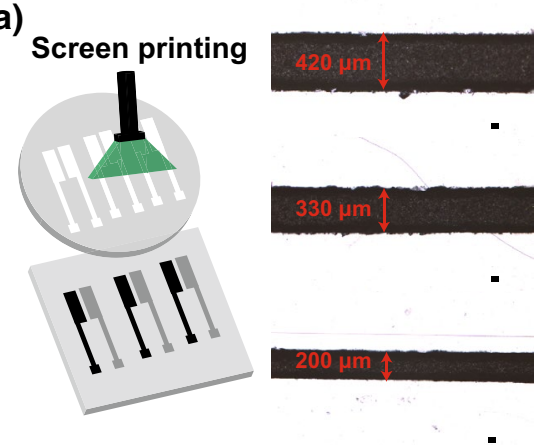

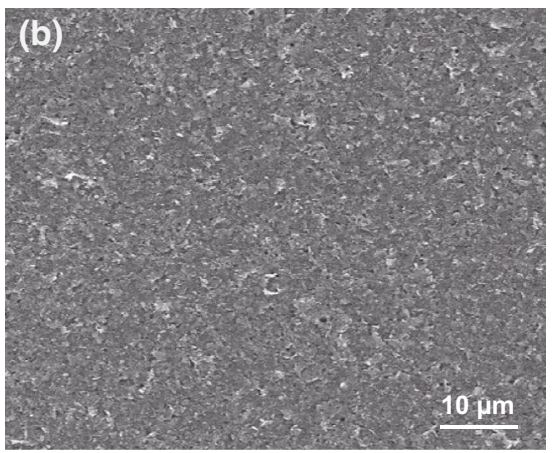
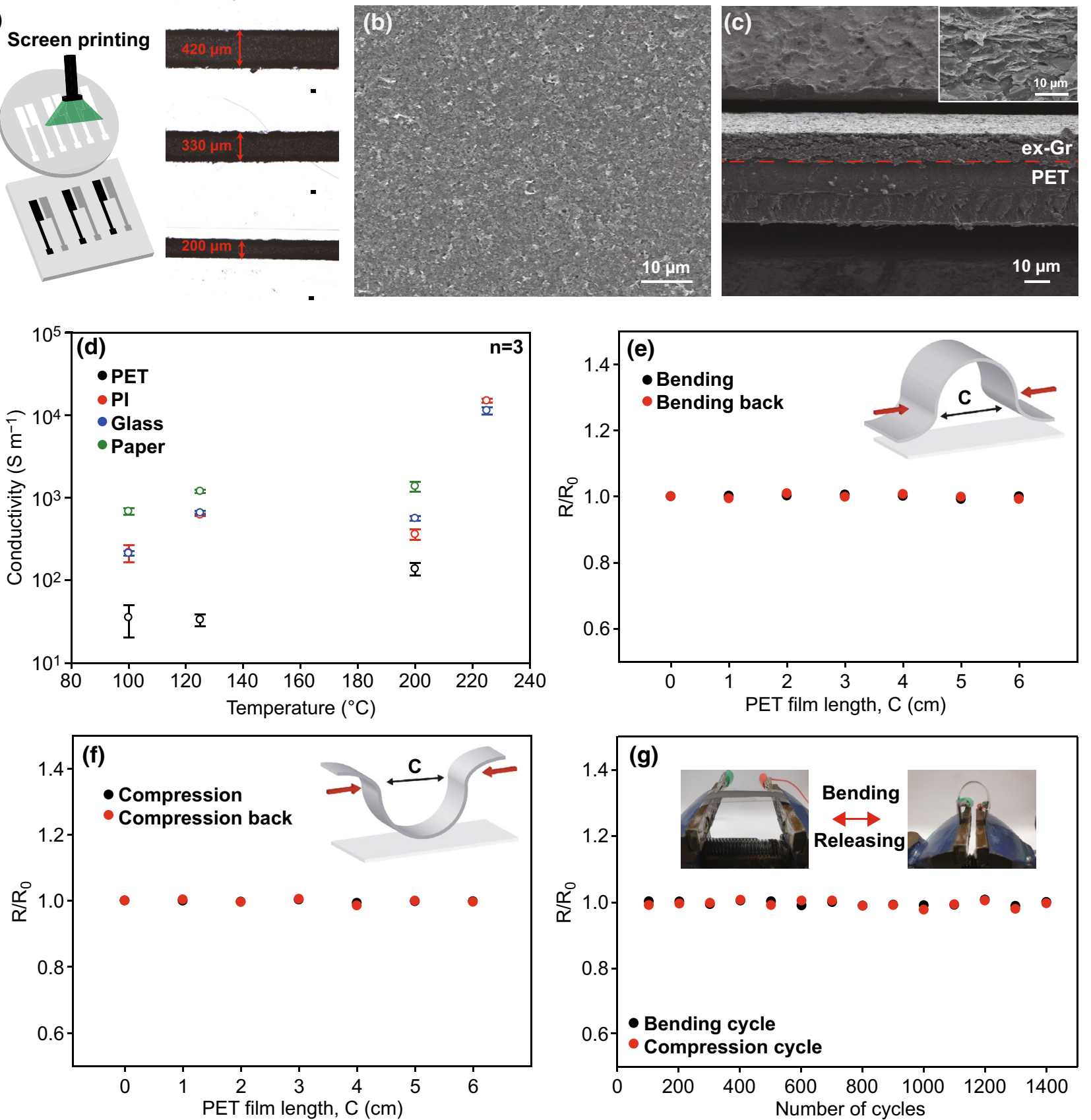

Fig. 3 a A schematic of screen-printing process using ex-Gr ink and optical microscope images of printed ex-Gr conductors at different resolutions $(200,330$, and $420 \mu \mathrm{m})$. b Surface and c cross-sectional SEM images of printed ex-Gr conductor (Inset: high-resolution SEM image of printed ex-Gr conductor). d Electrical conductivity of printed ex-Gr conductor (PET, PI, glass, and paper). e Bending and $\mathbf{f}$ compressing tests for evaluating resistance of different bending length. $\mathbf{g}$ Fatigue test for evaluating resistance of bending over 1400 cycles

influenced by the annealing temperature and film thickness (Figs. 3d and S3). The thermal annealing is a very efficient method to improve the conductivity of a graphene conductor, since it removes the impurities and solvents. In addition, degradation of binders occurs during annealing, resulting in a compactly interconnected graphene layer structure, which is responsible for high conductivity of the ex-Gr conductor [69]. When the annealing temperature was increased from 100 to $225^{\circ} \mathrm{C}$, the conductivity increased from $2.17 \times 10^{2}$ to $1.49 \times 10^{4} \mathrm{~S} \mathrm{~m}^{-1}$. The conductivity of the ex-Gr conductor 
based on a thermal annealing process is superior or comparable to other previous techniques, such as plasma and thermal annealing (Table S3). In addition, as the thickness decreased from 41 to $20 \mu \mathrm{m}$, the conductivity increased from $2.59 \times 10^{3}$ to $1.49 \times 10^{4} \mathrm{~S} \mathrm{~m}^{-1}$. Basis on these results, a high conductivity of $1.49 \times 10^{4} \mathrm{~S} \mathrm{~m}^{-1}$ was achieved at an annealing temperature of $225^{\circ} \mathrm{C}$ and an ex-Gr film thickness of $20 \mu \mathrm{m}$. This conductivity value for $\mathrm{Gr}$ ink is superior to other previously reported values for graphene-based conductors even with higher concentrations of graphene (Table S2). Further, the mechanical tolerance of the printed ex-Gr conductors on different substrates of PET, PI, and paper was investigated under bending and compressing. The change in the resistance of the ex-Gr conductor was measured at different bending and compressing states in forward and reverse directions (Figs. 3e, f and S4). A negligible change in the resistance was observed in these measurements for all printed ex-Gr conductors. The resistance of the ex-Gr electrode was further measured during 1400 bending-releasing and compressing-releasing cycles (Figs. $3 \mathrm{~g}$ and S4). The resistance remained stable, and the initial resistance was almost maintained during fatigue tests. Observation of
SEM images revealed that the ex-Gr conductor has a good adhesion at the interface of electrode and substrate with no mechanical damages after bending and fatigue tests (Fig. S5). The resistance tests demonstrate the combined properties of the ex-Gr conductor, i.e., its high conductivity and high mechanical stability.

\subsection{Electrochemical Performance of Screen-Printed $\mathrm{Na}^{+}$Sensor}

The highly conductive and flexible ex-Gr conductor can be used to fabricate flexible screen-printed electrochemical ion sensors. As an electrochemical ion sensor, a sodium ion selective membrane $\left(\mathrm{Na}^{+}\right.$-ISM), used to diagnose hyponatremia, was selected for the proof-of-concept testing. Ex-Gr and Ag inks were printed using a custom-designed stencil mask onto a highly flexible and thin PET substrate (Fig. 4a). The $\mathrm{Na}^{+}$-ISM was coated on the surface of the printed ex-Gr electrode serving as the sensing electrode. For a reference electrode, the $\mathrm{Ag} / \mathrm{AgCl}$ ink was printed onto the reaction part of the $\mathrm{Ag}$ circuit, followed by coating sodium (a)

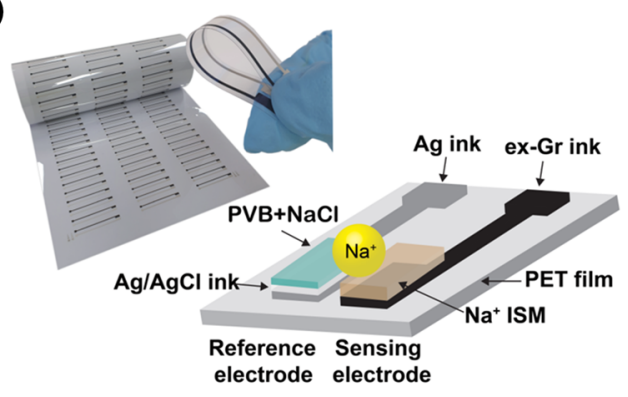

(d)

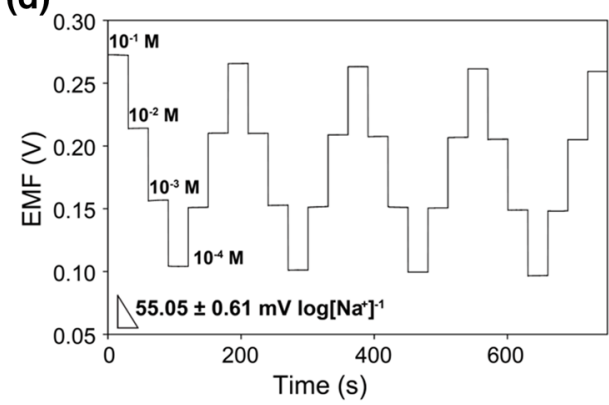

(b)

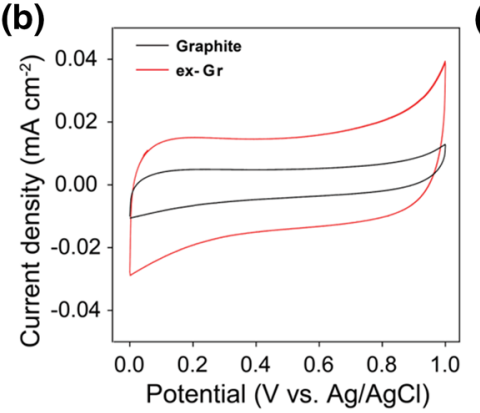

(e)

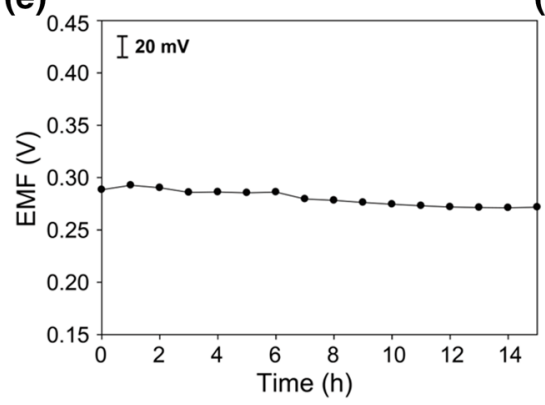

(c)

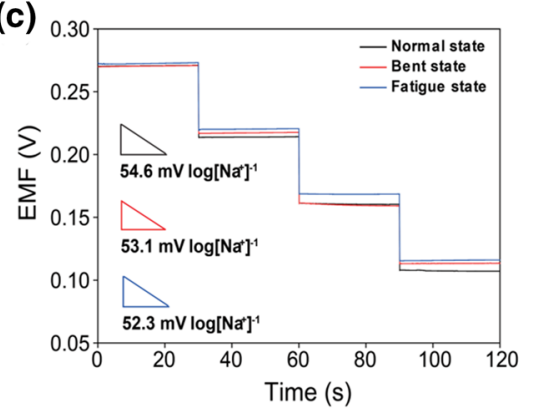

(f)

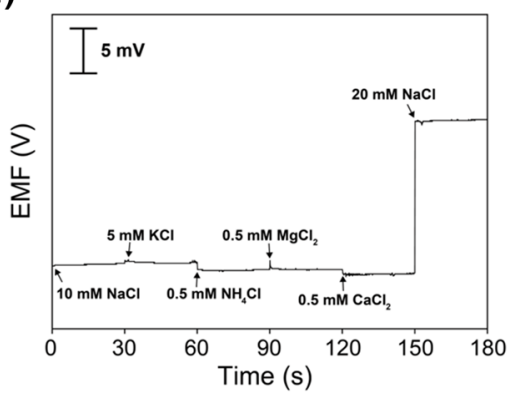

Fig. 4 a A photograph of screen-printed $\mathrm{Na}^{+}$sensors and a schematic of $\mathrm{Na}^{+}$-sensor structure. b CV curves of printed graphite and ex-Gr electrodes measured at a scan rate of $50 \mathrm{mV} \mathrm{s}^{-1}$ in $10^{-1} \mathrm{M} \mathrm{NaCl}$. c EMF responses of $\mathrm{Na}^{+}$sensors measured in the $10^{-1}-10^{-4} \mathrm{M} \mathrm{range}$ of $\mathrm{NaCl}$ concentration under mechanically normal and bent states and after fatigue test of 1400 bending and releasing cycles. $\mathbf{d}$ Repeatability test of $\mathrm{Na}^{+}$ sensor in various $\mathrm{NaCl}$ concentration solutions. e Long-term stability of $\mathrm{Na}^{+}$sensor measured for $15 \mathrm{~h}$ in $10^{-1} \mathrm{M} \mathrm{NaCl}$ solution. $\mathbf{f}$ Selectivity test of $\mathrm{Na}^{+}, \mathrm{K}^{+}, \mathrm{NH}_{4}^{+}, \mathrm{Ca}^{2+}, \mathrm{Mg}^{2+}$ ions 
chloride-containing polyvinyl butyral on the $\mathrm{Ag} / \mathrm{AgCl}-$ coated surface of the reference electrode. The cross section of the $\mathrm{Na}^{+}$sensor based on the two-electrode configuration is illustrated in Fig. 4a. It is worth noting that owing to the high surface area and electrical double-layer capacitance, the ex-Gr coating serves as an internal ion-to-electron transducer (also known as a solid contact) that facilitates the potentiometric performance. Compared to the graphite inkbased electrode, the ex-Gr electrode shows superior electrical double-layer capacitance and potential stability (Figs. 4b and S6). In addition, the ex-Gr electrode strongly resists the formation of a water layer between the ISM and electrode and is adequately insensitive to gas species (Fig. S7).

The potentiometric performance of the $\mathrm{Na}^{+}$sensor was evaluated by measuring the electromotive force (EMF) between the $\mathrm{Na}^{+}$-ISM and $\mathrm{Ag} / \mathrm{AgCl}$ electrodes using various $\mathrm{NaCl}$ solutions at physiologically relevant concentrations. The calibration curve of the EMF versus $\mathrm{Na}^{+}$concentration is also depicted in Fig. S8. The $\mathrm{Na}^{+}$sensor shows an average sensitivity of $54.0 \mathrm{mV}$ per decade of $\mathrm{Na}^{+}$concentration $(n=5)$ with a relative standard deviation (RSD) of $1.2 \%$. This average sensitivity indicates the near-Nernstian behavior of the device. The small standard deviation of the slope $\left( \pm 0.65 \mathrm{mV} \log \left[\mathrm{Na}^{+}\right]^{-1}\right)$ and absolute potential $( \pm 7.9 \mathrm{mV})$ from five sensors demonstrate the good reproducibility of the $\mathrm{Na}^{+}$sensor fabricated by the screen-printing process. The $\mathrm{Na}^{+}$sensor exhibits a rapid response time of $3.6 \mathrm{~s}$, as measured by successively increasing the $\mathrm{Na}^{+}$concentration from 100 to $1 \mathrm{mM}$ (Fig. S9). This fast response time is important for the real-time monitoring of sweat, because the sensor would allow quick and accurate assessment of changes in the $\mathrm{Na}^{+}$concentration. Based on the intersection of two slopes of EMF versus concentration sodium ion (Fig. $\mathrm{S} 10)$, the detection limit of the $\mathrm{Na}^{+}$sensor was calculated to be $14.8 \mu \mathrm{M}$. The effect of annealing temperature on the performance of the $\mathrm{Na}^{+}$sensor was investigated by measuring the sensitivity with different annealing temperatures of $60,100,125$, and $200{ }^{\circ} \mathrm{C}$ (Fig. S11). The $\mathrm{Na}^{+}$sensors exhibited an average sensitivity of $53.1 \pm 2.7 \mathrm{mV} \log \left[\mathrm{Na}^{+}\right]^{-1}$. The small standard deviation indicates no effect of annealing temperature on the electrochemical sensing performance of the $\mathrm{Na}^{+}$sensor. The sensitivity of the $\mathrm{Na}^{+}$sensor was further investigated in a mechanically bent state (Fig. 4c). Remarkably, the $\mathrm{Na}^{+}$sensor exhibits a slope of $53.1 \mathrm{mV} \log \left[\mathrm{Na}^{+}\right]^{-1}$ $\left(R^{2}=0.9977\right)$, which is consistent with the response of the same sensor in the normal state. After a fatigue test of 1,400 bending and releasing cycles, a high sensitivity of $52.3 \mathrm{mV}$ $\log \left[\mathrm{Na}^{+}\right]^{-1}\left(R^{2}=0.9999\right)$ for the $\mathrm{Na}^{+}$sensor was maintained (Fig. 4c). This high sensing performance of the $\mathrm{Na}^{+}$sensor against mechanical stress is attributed to the unique mechanical strength of the ex-Gr sheets and EC binder. The electrochemical stability of the $\mathrm{Na}^{+}$sensor was also evaluated by a repeatability test. Figure $4 d$ shows the dynamic EMF response of the $\mathrm{Na}^{+}$sensor under the repeated sequential loading of different $\mathrm{NaCl}$ solutions in the concentration range $0.1-100 \mathrm{mM}$. During this measurement, the original EMF responses of the $\mathrm{Na}^{+}$sensor were retained without any hysteresis effects. After five consecutive repetitions, the $\mathrm{Na}^{+}$ sensor exhibited a sensitivity of $54.4 \mathrm{mV} \log \left[\mathrm{Na}^{+}\right]^{-1}$, which is very similar to the initial value of $56.1 \mathrm{mV} \log \left[\mathrm{Na}^{+}\right]^{-1}$, indicating excellent repeatability. To demonstrate reusability, the sensitivity of the $\mathrm{Na}^{+}$sensor was evaluated repeatedly by measuring EMF responses and then washing with DI water. After 10 cycles of repeating tests, the $\mathrm{Na}^{+}$sensor still exhibited a high sensitivity of $56.3 \mathrm{mV} \log \left[\mathrm{Na}^{+}\right]^{-1}$, which is very close to the initial sensitivity of $55.4 \mathrm{mV} \log \left[\mathrm{Na}^{+}\right]^{-1}$ (Fig. S12). This indicates good reusability of the $\mathrm{Na}^{+}$sensor.

Further, potentiometric measurements were performed to investigate the durability and selectivity of the $\mathrm{Na}^{+}$sensor. Its durability was investigated by evaluating the potential drift over a period of $15 \mathrm{~h}$ (Fig. 4e). The $\mathrm{Na}^{+}$sensor exhibits a potential drift of $1.2 \mathrm{mV} \mathrm{h}^{-1}$, which is considerably lower than that of other $\mathrm{Na}^{+}$sensors based on other carbon or gold electrodes [70-72]. The selectivity of the $\mathrm{Na}^{+}$sensor was investigated by determining the selectivity coefficients of the $\mathrm{Na}^{+}$sensor for different interfering ions using the separatesolution method. The selectivity coefficients for $\mathrm{K}^{+}, \mathrm{NH}_{4}{ }^{+}$, $\mathrm{Ca}^{2+}$, and $\mathrm{Mg}^{2+}$ were found to be less than 1 (Table S4), indicating good ion selectivity of the $\mathrm{Na}^{+}$sensor. To further demonstrate high selectivity of the $\mathrm{Na}^{+}$sensor, the change in EMF response was measured by adding interfering ions such as $5 \mathrm{mM} \mathrm{KCl}, 0.5 \mathrm{mM} \mathrm{NH}_{4} \mathrm{Cl}, 0.5 \mathrm{mM} \mathrm{MgCl}_{2}$, and $0.5 \mathrm{mM}$ $\mathrm{CaCl}_{2}$ to a $10 \mathrm{mM} \mathrm{NaCl}$ solution (Fig. $4 \mathrm{f}$ ). The interfering ion concentrations were selected considering physiological concentrations in human sweat. The sensor showed no response to the addition of such ions. After the addition of a $10 \mathrm{mM} \mathrm{NaCl}$ solution, the EMF signal increased according to Nernstian behavior. To further investigate the effect of $\mathrm{pH}$ on the sensitivity of the $\mathrm{Na}^{+}$sensor, the sensitivity was evaluated by measuring EMF response at different $\mathrm{pH}$ values of $4.50,6.24$, and 8.53, decreasing the concentration of the $\mathrm{Na}^{+}$solution (Fig. S13). The EMF signals and sensitivities 
almost overlapped at the $\mathrm{pH}$ levels, indicating no interference of $\mathrm{pH}$ with the sensor performance of the $\mathrm{Na}^{+}$sensor.

\subsection{On-Body Test of Sodium Ion Sensor}

To demonstrate real-time monitoring of sodium ions in human sweat with a screen-printed $\mathrm{Na}^{+}$sensor based on ex-Gr ink, a wristwatch-type wearable device was fabricated by connecting the $\mathrm{Na}^{+}$sensor and a PCB containing a Bluetooth module (Fig. 5a). The data collected continuously from the PCB were wirelessly transmitted to a smartphone application. To ensure accurate signal collection by the on-body test, the EMF-to-concentration calibration of the sweat sensor was confirmed before and after the on-body measurements (Fig. 5b). Figure 5c shows the on-body test condition with the subject wearing the experimental sweat watch sensor while riding a stationary exercise bike at room temperature (Movie S1). The maximum exercise power was
$700 \mathrm{~W}$ and the total exercise time was $20 \mathrm{~min}$, and the average heart rate of the subject was $108 \mathrm{bpm}$. The profiles of EMF and $\mathrm{Na}^{+}$concentration collected during the real-time on-body sweat test are displayed in Fig. 5c. The collected EMF signals were converted to $\mathrm{Na}^{+}$concentration based on a calibration curve obtained before the on-body test. When the subject started cycling, there was significant signal noise observed due to an insufficient amount of sweat for a signal from the two-electrode configuration. After approximately $370 \mathrm{~s}, \mathrm{EMF}$ signals and corresponding $\mathrm{Na}^{+}$concentrations rapidly changed, indicating that there was sufficient sweat collected for the two-electrode sensor. This phenomenon was also observed for previously reported electrochemical ion sensors [73-76] and is related to the perspiration rate of the subject while exercising. The $\mathrm{Na}^{+}$concentration gradually stabilized, reaching approximately $24 \mathrm{mM}$, which is the physiological $\mathrm{Na}^{+}$ion concentration. The resultant real-time data of the $\mathrm{Na}^{+}$sensor are similar to those obtained in previously reported on-body tests [71, 77-79].
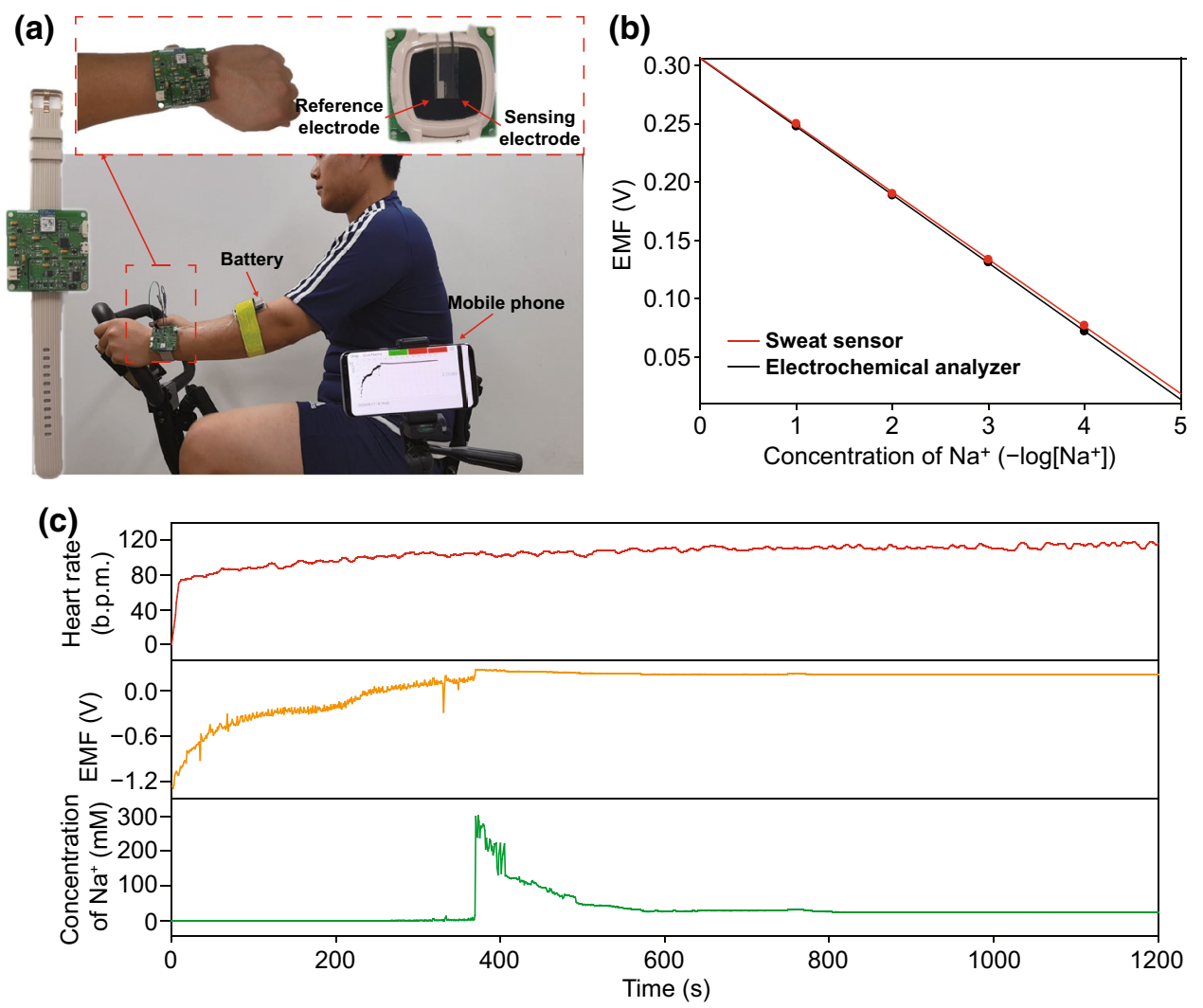

Fig. 5 a A photographs of wearing wristwatch-type wearable device for real-time analysis during stationary cycling. b Calibration curves of electrochemical analyzer and sweat sensor. c Subject's simultaneous heart rate, EMF and converted concentration of $\mathrm{Na}^{+}$during stationary cycling 


\section{Conclusions}

We have successfully demonstrated a scalable and efficient fluid dynamics process for the preparation of high-concentration conductive inks from defect-free ex-Gr with high electrical conductivity. To date, the preparation of graphenebased inks has suffered from a lack of mass production and a low concentration of graphene suspensions. The TC flowinduced process enabled an efficient exfoliation of graphite into defect-free graphene nanosheets in a high yield of $53.5 \%$ and a high concentration of $47.5 \mathrm{mg} \mathrm{mL}^{-1}$, which are superior to other solution-based methods. The ultrahigh concentration of the ex-Gr ink allowed us to eliminate the post-evaporation step and additional binders that are often required for the screen-printing process. The maximum graphite loading was $200 \mathrm{mg} \mathrm{mL}^{-1}$ for the fluid dynamics process owing to the high viscosity of the graphite loading in the limited space of our fluidic reactor. However, the fluidic reactor can be easily scaled up, and thus, it is possible that a larger fluidic reactor can process graphite loading over $200 \mathrm{mg} \mathrm{mL}^{-1}$. The as-obtained ex-Gr ink facilitated the screen-printing process, which was used to fabricate a high-performance electro-mechanical ex-Gr conductor. The ex-Gr conductor exhibited a high electrical conductivity of $1.49 \times 10^{4} \mathrm{~S} \mathrm{~m}^{-1}$ and low resistance variation in mechanical bending and fatigue tests. Using the ex-Gr ink with these attractive merits, potentiometric $\mathrm{Na}^{+}$sensors based on a twoelectrode configuration were fabricated. The $\mathrm{Na}^{+}$sensors showed rapid response times and high sensitivities in the mechanically normal and bent states with good repeatability and selectivity. Furthermore, a wristwatch-type $\mathrm{Na}^{+}$sensor was fabricated by integrating it with a wireless electronic module. A successful on-body test was performed by the real-time wireless monitoring of the $\mathrm{Na}^{+}$concentration of the sweat of a volunteer during an indoor cycling exercise. The wearable sweat sensor could be further improved by integrating temperature and other ion sensors (e.g., $\mathrm{K}^{+}, \mathrm{pH}$, and $\mathrm{Cl}^{-}$) to analyze multiple analytes in sweat, and thus for real-time monitoring of the physiological status of individuals. The highly conductive and highly concentrated graphene ink presented in this work opens a promising opportunity for the fabrication of low-cost, scalable, and high-performance electrochemical ion sensors for the practical real-time monitoring of human health.
Acknowledgements D.S.K., J.-M.J., and H.J.P. have contributed equally to this work. This work was supported by the National Research Foundation of Korea (NRF) Grant funded by the Ministry of Science and ICT (No. 2021R1A2C1009926). This research has been performed as a cooperation project of "Basic project (referring to projects performed with the budget directly contributed by the Government to achieve the purposes of establishment of Government-funded research Institutes)" and supported by the KOREA RESEARCH INSTITUTE of CHEMICAL TECHNOLOGY (KRICT) (SS2042-10). This research was supported by Basic research project (Project: 21-3212-1) of the Korea institute of Geoscience and Mineral resources funded by the Ministry of Science and ICT of Korea. This work was also funded by Nanomedical Devices Development Project of NNFC in 2021.

Open Access This article is licensed under a Creative Commons Attribution 4.0 International License, which permits use, sharing, adaptation, distribution and reproduction in any medium or format, as long as you give appropriate credit to the original author(s) and the source, provide a link to the Creative Commons licence, and indicate if changes were made. The images or other third party material in this article are included in the article's Creative Commons licence, unless indicated otherwise in a credit line to the material. If material is not included in the article's Creative Commons licence and your intended use is not permitted by statutory regulation or exceeds the permitted use, you will need to obtain permission directly from the copyright holder. To view a copy of this licence, visit http://creativecommons.org/licenses/by/4.0/.

Supplementary Information The online version contains supplementary material available at https://doi.org/10.1007/s4082 0-021-00617-3.

\section{References}

1. H. Teymourian, M. Parrilla, J.R. Sempionatto, N.F. Montiel, A. Barfidokht et al., Wearable electrochemical sensors for the monitoring and screening of drugs. ACS Sen. 5, 2679-2700 (2020). https://doi.org/10.1021/acssensors.0c01318

2. J. Kim, A.S. Campbell, B.E.-F. Ávila, J. Wang, Wearable biosensors for healthcare monitoring. Nat. Biotechnol. 37, 389-406 (2019). https://doi.org/10.1038/s41587-019-0045-y

3. Y. Song, J. Min, W. Gao, Wearable and implantable electronics: moving toward precision therapy. ACS Nano 13, 12280 12286 (2019). https://doi.org/10.1021/acsnano.9b08323

4. J. Min, J.R. Sempionatto, H. Teymourian, J. Wang, W. Gao, Wearable electrochemical biosensors in north america. Biosens. Bioelectron. 172, 112750 (2021). https://doi. org/10.1016/j.bios.2020.112750

5. M. Bariya, L. Li, R. Ghattamaneni, C.H. Ahn, H.Y.Y. Nyein et al., Glove-based sensors for multimodal monitoring of natural sweat. Sci. Adv. 6, eabb8308 (2020). https://doi. org/10.1126/sciadv.abb8308 
6. Y. Ling, T. An, L.W. Yap, B. Zhu, S. Gong et al., Disruptive, soft, wearable sensors. Adv. Mater. 32, 1904664 (2020). https ://doi.org/10.1002/adma.201904664

7. Y. Yu, H.Y.Y. Nyein, W. Gao, A. Javey, Flexible electrochemical bioelectronics: the rise of in situ bioanalysis. Adv. Mater. 32, 1902083 (2020). https://doi.org/10.1002/adma.201902083

8. T. Li, Y. Li, T. Zhang, Materials, structures, and functions for flexible and stretchable biomimetic sensors. Acc. Chem. Res. 52, 288-296 (2019). https://doi.org/10.1021/acs.accou nts. 8 b00497

9. Z. Lou, L. Wang, K. Jiang, Z. Wei, G. Shen, Reviews of wearable healthcare systems: materials, devices and system integration. Mater. Sci. Eng. R Rep. 140, 100523 (2020). https://doi.org/10.1016/j.mser.2019.100523

10. Y. Shen, Y. Wang, Z. Luo, B. Wang, Durable, sensitive, and wide-range wearable pressure sensors based on wavystructured flexible conductive composite film. Macromol. Mater. Eng. 305, 2000206 (2020). https://doi.org/10.1002/ mame. 202000206

11. J. Yun, H. Lee, C. Song, Y.R. Jeong, J.W. Park et al., A fractal-designed stretchable and transparent microsupercapacitor as a skin-attachable energy storage device. Chem. Eng. J. 387, 124076 (2020). https://doi.org/10.1016/j. cej.2020.124076

12. T. Chu, J. Chu, B. Gao, B. He, Modern evolution of paperbased analytical devices for wearable use: from disorder to order. Analyst 145, 5388-5399 (2020). https://doi. org/10.1039/D0AN00994F

13. H.C. Lee, E.Y. Hsieh, K. Yong, S. Nam, Multiaxiallystretchable kirigami-patterned mesh design for graphene sensor devices. Nano Res. 13, 1406-1412 (2020). https:// doi.org/10.1007/s12274-020-2662-7

14. S. Wang, Y. Bai, X. Yang, L. Liu, L. Li et al., Highly stretchable potentiometric ion sensor based on surface strain redistributed fiber for sweat monitoring. Talanta 214, 120869 (2020). https://doi.org/10.1016/j.talanta.2020.120869

15. H. Teymourian, A. Barfidokht, J. Wang, Electrochemical glucose sensors in diabetes management: an updated review (2010-2020). Chem. Soc. Rev. 49, 7671-7709 (2020). https ://doi.org/10.1039/D0CS00304B

16. S.P. Sreenilayam, I.U. Ahad, V. Nicolosi, V.A. Garzon, D. Brabazon, Advanced materials of printed wearables for physiological parameter monitoring. Mater. Today 32, 147177 (2020). https://doi.org/10.1016/j.mattod.2019.08.005

17. H. Lee, Y.J. Hong, S. Baik, T. Hyeon, D.-H. Kim, Enzymebased glucose sensor: from invasive to wearable device. Adv. Healthc. Mater. 7, 1701150 (2018). https://doi.org/10.1002/ adhm.201701150

18. Y. Yan, W. Gao, Wearable and flexible electronics for continuous molecular monitoring. Chem. Soc. Rev. 48, 1465-1491 (2019). https://doi.org/10.1039/C7CS00730B

19. A.J. Bandodkar, J. Wang, Non-invasive wearable electrochemical sensors-a review. Trends Biotechnol. 32, 363371 (2014). https://doi.org/10.1016/j.tibtech.2014.04.005
20. M.A. Arnold, G.W. Small, Noninvasive glucose sensing. Anal. Chem. 77, 5429-5439 (2005). https://doi.org/10.1021/ ac050429e

21. J. Heikenfeld, A. Jajack, J. Rogers, P. Gutruf, L. Tian et al., Wearable sensors: modalities, challenges, and prospects. Lab Chip 18, 217-248 (2018). https://doi.org/10.1039/ C7LC00914C

22. R.M. Torrente-Rodríguez, J. Tu, Y. Yang, J. Min, M. Wang et al., Investigation of cortisol dynamics in human sweat using a graphene-based wireless mHealth system. Matter 2, 921-937 (2020). https://doi.org/10.1016/j.matt.2020.01.021

23. J. Xu, Z. Zhang, S. Gan, H. Gao, H. Kong et al., Highlystretchable fiber-based potentiometric ion sensors for multichannel real-time analysis of human sweat. ACS Sens. 5, 2834-2842 (2020). https://doi.org/10.1021/acssensors.0c009 60

24. Q. An, S. Gan, J. Xu, Y. Bao, T. Wu et al., A multichannel electrochemical all-solid-state wearable potentiometric sensor for real-time sweat ion monitoring. Electrochem. Commun. 107, 106553 (2019). https://doi.org/10.1016/j.eleco m.2019.106553

25. H.Y.Y. Nyein, M. Bariya, L. Kivimäki, S. Uusitalo, T.S. Liaw et al., Regional and correlative sweat analysis using high-throughput microfluidic sensing patches toward decoding sweat. Sci. Adv. 5, eaaw9906 (2019). https://doi. org/10.1126/sciadv.aaw9906

26. H.Y.Y. Nyein, L.-C. Tai, Q.P. Ngo, M. Chao, G.B. Zhang et al., A wearable microfluidic sensing patch for dynamic sweat secretion analysis. ACS Sens. 3, 944-952 (2018). https://doi.org/10.1021/acssensors.7b00961

27. S. Zhang, M.A. Zahed, Md. Sharifuzzaman, S. Yoon, $\mathrm{X}$. Hui et al., A wearable battery-free wireless and skininterfaced microfluidics integrated electrochemical sensing patch for on-site biomarkers monitoring in human perspiration. Biosens. Bioelectron. 175, 112844 (2021). https://doi. org/10.1016/j.bios.2020.112844

28. M. Ochoa, R. Rahimi, J. Zhou, H. Jiang, C.K. Yoon et al., Integrated sensing and delivery of oxygen for next-generation smart wound dressings. Microsyst. Nanoeng. 6, 46 (2020). https://doi.org/10.1038/s41378-020-0141-7

29. S. Bobovych, F. Sayeed, N. Banerjee, R. Robucci, R.P. Allen, RestEaZe: low-power accurate sleep monitoring using a wearable multi-sensor ankle band. Smart Health 16, 100113 (2020). https://doi.org/10.1016/j.smhl.2020.100113

30. L. Manjakkal, W. Dang, N. Yogeswaran, R. Dahiya, Textile-based potentiometric electrochemical $\mathrm{pH}$ sensor for wearable applications. Biosensors 9, 14 (2019). https://doi. org/10.3390/bios9010014

31. A. Hatamie, S. Angizi, S. Kumar, C.M. Pandey, A. Simchi et al., Textile based chemical and physical sensors for healthcare monitoring. J. Electrochem. Soc. 167, 037546 (2020). https://doi.org/10.1149/1945-7111/ab6827

32. W. He, C. Wang, H. Wang, M. Jian, W. Lu et al., Integrated textile sensor patch for real-time and multiplex sweat analysis. Sci. Adv. 5, eaax0649 (2019). https://doi.org/10.1126/ sciadv.aax0649 
33. A.J. Bandodkar, W. Jia, C. Yardimcı, X. Wang, J. Ramirez et al., Tattoo-based noninvasive glucose monitoring: a proof-of-concept study. Anal. Chem. 87, 394-398 (2015). https://doi.org/10.1021/ac504300n

34. J.R. Sempionatto, I. Jeerapan, S. Krishnan, J. Wang, Wearable chemical sensors: emerging systems for on-body analytical chemistry. Anal. Chem. 92, 378-396 (2020). https:// doi.org/10.1021/acs.analchem.9b04668

35. A.J. Bandodkar, I. Jeerapan, J. Wang, Wearable chemical sensors: present challenges and future prospects. ACS Sens. 1, 464-482 (2016). https://doi.org/10.1021/acssensors.6b00250

36. T.R. Ray, J. Choi, A.J. Bandodkar, S. Krishnan, P. Gutruf et al., Bio-integrated wearable systems: a comprehensive review. Chem. Rev. 119, 5461-5533 (2019). https://doi. org/10.1021/acs.chemrev.8b00573

37. Q. Li, J. Zhang, Q. Li, G. Li, X. Tian et al., Review of printed electrodes for flexible devices. Front. Mater. 5, 77 (2019). https://doi.org/10.3389/fmats.2018.00077

38. P.S. Sfragano, S. Laschi, I. Palchetti, Sustainable printed electrochemical platforms for greener analytics. Front. Chem. 8, 644 (2020). https://doi.org/10.3389/fchem.2020.00644

39. F. Bonaccorso, A. Bartolotta, J.N. Coleman, C. Backes, 2D-crystal-based functional inks. Adv. Mater. 28, 6136-6166 (2016). https://doi.org/10.1002/adma.201506410

40. G. Hu, J. Kang, L.W.T. Ng, X. Zhu, R.C.T. Howe et al., Functional inks and printing of two-dimensional materials. Chem. Soc. Rev. 47, 3265-3300 (2018). https://doi.org/10.1039/ C8CS00084K

41. T.S. Tran, N.K. Dutta, N.R. Choudhury, Graphene inks for printed flexible electronics: graphene dispersions, ink formulations, printing techniques and applications. Adv. Colloid Interface Sci. 261, 41-61 (2018). https://doi.org/10.1016/j. cis.2018.09.003

42. K. Pan, Y. Fan, T. Leng, J. Li, Z. Xin et al., Sustainable production of highly conductive multilayer graphene ink for wireless connectivity and IoT applications. Nat. Commun. 9, 5197 (2018). https://doi.org/10.1038/s41467-018-07632-w

43. S. Park, R.S. Ruoff, Chemical methods for the production of graphenes. Nat. Nanotechnol. 4, 217-224 (2009). https://doi. org/10.1038/nnano.2009.58

44. V. Nicolosi, M. Chhowalla, M.G. Kanatzidis, M.S. Strano, J.N. Coleman, Liquid exfoliation of layered materials. Science 340, 1226419 (2013). https://doi.org/10.1126/science.1226419

45. S. Mao, H. Pu, J. Chen, Graphene oxide and its reduction: modeling and experimental progress. RSC Adv. 2, 2643-2662 (2012). https://doi.org/10.1039/C2RA00663D

46. K.R. Paton, E. Varrla, C. Backes, R.J. Smith, U. Khan et al., Scalable production of large quantities of defect-free fewlayer graphene by shear exfoliation in liquids. Nat. Mater. 13, 624-630 (2014). https://doi.org/10.1038/nmat3944

47. S. Bellani, E. Petroni, A.E. Del Rio Castillo, N. Curreli, B. Martín-García et al., Scalable production of graphene inks via wet-jet milling exfoliation for screen-printed micro-supercapacitors. Adv. Funct. Mater. 29, 1807659 (2019). https://doi. org/10.1002/adfm.201807659
48. Y. Shin, S. Vranic, X. Just-Baringo, S.M. Gali, T. Kisby et al., Stable, concentrated, biocompatible, and defect-free graphene dispersions with positive charge. Nanoscale 12, 12383-12394 (2020). https://doi.org/10.1039/D0NR02689A

49. F. Tehrani, M. Beltrán-Gastélum, K. Sheth, A. Karajic, L. Yin et al., Laser-induced graphene composites for printed, stretchable, and wearable electronics. Adv. Mater. Technol. 4, 1900162 (2019). https://doi.org/10.1002/admt.201900162

50. N. Wei, L. Yu, Z. Sun, Y. Song, M. Wang et al., Scalable salt-templated synthesis of nitrogen-doped graphene nanosheets toward printable energy storage. ACS Nano 13, 7517-7526 (2019). https://doi.org/10.1021/acsnano.9b031 57

51. M. Yi, Z. Shen, A review on mechanical exfoliation for the scalable production of graphene. J. Mater. Chem. A 3, 11700 11715 (2015). https://doi.org/10.1039/C5TA00252D

52. M. Lotya, P.J. King, U. Khan, S. De, J.N. Coleman, Highconcentration, surfactant-stabilized graphene dispersions. ACS Nano 4, 3155-3162 (2010). https://doi.org/10.1021/ nn1005304

53. F. Torrisi, T. Hasan, W. Wu, Z. Sun, A. Lombardo et al., Inkjet-printed graphene electronics. ACS Nano 6, 2992-3006 (2012). https://doi.org/10.1021/nn2044609

54. A.V. Tyurnina, I. Tzanakis, J. Morton, J. Mi, K. Porfyrakis et al., Ultrasonic exfoliation of graphene in water: a key parameter study. Carbon 168, 737-747 (2020). https://doi. org/10.1016/j.carbon.2020.06.029

55. K. Manna, L. Wang, K.J. Loh, W.-H. Chiang, Printed strain sensors using graphene nanosheets prepared by water-assisted liquid phase exfoliation. Adv. Mater. Interfaces 6, 1900034 (2019). https://doi.org/10.1002/admi.201900034

56. T.S. Tran, S.J. Park, S.S. Yoo, T.-R. Lee, T. Kim, High shearinduced exfoliation of graphite into high quality graphene by Taylor-Couette flow. RSC Adv. 6, 12003-12008 (2016). https ://doi.org/10.1039/C5RA22273G

57. J.-M. Jeong, S.B. Jin, H.J. Park, S.H. Park, H. Jeon et al., Large-scale fast fluid dynamic processes for the syntheses of 2D nanohybrids of metal nanoparticle-deposited boron nitride nanosheet and their glycolysis of poly(ethylene terephthalate). Adv. Mater. Interfaces 7, 2000599 (2020). https://doi. org/10.1002/admi.202000599

58. J.H. Yoon, S.-M. Kim, Y. Eom, J.M. Koo, H.-W. Cho et al., Extremely fast self-healable bio-based supramolecular polymer for wearable real-time sweat-monitoring sensor. ACS Appl. Mater. Interfaces 11, 46165-46175 (2019). https://doi. org/10.1021/acsami.9b16829

59. J.H. Yoon, S.-M. Kim, H.J. Park, Y.K. Kim, D.X. Oh et al., Highly self-healable and flexible cable-type $\mathrm{pH}$ sensors for real-time monitoring of human fluids. Biosnes. Bioelectron. 150, 111946 (2020). https://doi.org/10.1016/j.bios.2019.11194 6

60. J.-M. Jeong, H.G. Kang, H.-J. Kim, S.B. Hong, H. Jeon et al., Hydraulic power manufacturing for highly scalable and stable 2D nanosheet dispersions and their film electrode application. Adv. Funct. Mater. 28, 1802952 (2018). https://doi. org/10.1002/adfm.201802952 
61. J.A. Robinson, C.P. Puls, N.E. Staley, J.P. Stitt, M.A. Fanton et al., Raman topography and strain uniformity of large-area epitaxial graphene. Nano Lett. 9, 964-968 (2009). https://doi. org/10.1021/nl802852p

62. Y. Hao, Y. Wang, L. Wang, Z. Ni, Z. Wang et al., Probing layer number and stacking order of few-layer graphene by raman spectroscopy. Small 6, 195-200 (2010). https://doi. org/10.1002/smll.200901173

63. C.V. Gomez, T. Tene, M. Guevara, G.T. Usca, D. Colcha et al., Preparation of few-layer graphene dispersions from hydrothermally expanded graphite. Appl. Sci. 9, 2539 (2019). https:// doi.org/10.3390/app9122539

64. N. Karim, M. Zhang, S. Afroj, V. Koncherry, P. Potluri et al., Graphene-based surface heater for de-icing applications. RSC Adv. 8, 16815-16823 (2018). https://doi.org/10.1039/C8RA0 $2567 \mathrm{C}$

65. S. Afroj, S. Tan, A.M. Abdelkader, K.S. Novoselov, N. Karim, Highly conductive, scalable, and machine washable graphenebased e-textiles for multifunctional wearable electronic applications. Adv. Funct. Mater. 30, 2000293 (2020). https://doi. org/10.1002/adfm.202000293

66. H. Jeon, J.-M. Jeong, H.G. Kang, H.-J. Kim, J. Park et al., Scalable water-based production of highly conductive 2D nanosheets with ultrahigh volumetric capacitance and rate capability. Adv. Energy Mater. 8, 1800227 (2018). https://doi. org/10.1002/aenm.201800227

67. S.B. Hong, J.-M. Jeong, H.G. Kang, D. Seo, Y. Cha et al., Fast and scalable hydrodynamic synthesis of $\mathrm{MnO}_{2} /$ defectfree graphene nanocomposites with high rate capability and long cycle life. ACS Appl. Mater. Interfaces 10, 35250-35259 (2018). https://doi.org/10.1021/acsami.8b12894

68. B. Alfano, T. Polichetti, M. Mauriello, M.L. Miglietta, F. Ricciardella et al., Modulating the sensing properties of graphene through an eco-friendly metal-decoration process. Sens. Actuat. B 222, 1032-1042 (2016). https://doi.org/10.1016/j. snb.2015.09.008

69. K. Arapov, G. Bex, R. Hendriks, E. Rubingh, R. Abbel et al., Conductivity enhancement of binder-based graphene inks by photonic annealing and subsequent compression rolling. Adv. Eng. Mater. 18, 1234-1239 (2016). https://doi.org/10.1002/ adem. 201500646
70. S. Roy, M. David-Pur, Y. Hanein, Carbon nanotube-based ion selective sensors for wearable applications. ACS Appl. Mater. Interfaces 9, 35169-35177 (2017). https://doi.org/10.1021/ acsami. $7 b 07346$

71. A.J. Bandodkar, D. Molinnus, O. Mirza, T. Guinovart, J.R. Windmiller et al., Epidermal tattoo potentiometric sodium sensors with wireless signal transduction for continuous noninvasive sweat monitoring. Biosens. Bioelectron. 54, 603-609 (2014). https://doi.org/10.1016/j.bios.2013.11.039

72. W. Gao, S. Emaminejad, H.Y.Y. Nyein, S. Challa, K. Chen et al., Fully integrated wearable sensor arrays for multiplexed in situ perspiration analysis. Nature 529, 509-514 (2016). https://doi.org/10.1038/nature16521

73. A. Alizadeh, A. Burns, R. Lenigk, R. Gettings, J. Ashe et al., A wearable patch for continuous monitoring of sweat electrolytes during exertion. Lab Chip 18, 2632-2641 (2018). https://doi. org/10.1039/C8LC00510A

74. M. McCaul, A. Porter, R. Barrett, P. White, F. Stroiescu et al., Wearable platform for real-time monitoring of sodium in sweat. ChemPhysChem 19, 1531-1536 (2018). https://doi. org/10.1002/cphc.201701312

75. G. Liu, C. Ho, N. Slappey, Z. Zhou, S.E. Snelgrove et al., A wearable conductivity sensor for wireless real-time sweat monitoring. Sens. Actuat. B 227, 35-42 (2016). https://doi. org/10.1016/j.snb.2015.12.034

76. B. Ma, J. Chi, C. Xu, Y. Ni, C. Zhao et al., Wearable capillary microfluidics for continuous perspiration sensing. Talanta $\mathbf{2 1 2}$, 120786 (2020). https://doi.org/10.1016/j.talanta.2020.120786

77. M. Parrilla, I. Ortiz-Gómez, R. Cánovas, A. Salinas-Castillo, M. Cuartero et al., Wearable potentiometric ion patch for onbody electrolyte monitoring in sweat: toward a validation strategy to ensure physiological relevance. Anal. Chem. 91, 86448651 (2019). https://doi.org/10.1021/acs.analchem.9b02126

78. T. Glennon, C. O’Quigley, M. McCaul, G. Matzeu, S. Beirne et al., "Sweatch": a wearable platform for harvesting and analysing sweat sodium content. Electroanalysis 28, 1283-1289 (2016). https://doi.org/10.1002/elan.201600106

79. G. Matzeu, C. O’Quigley, E. McNamara, C. Zuliani, C. Fay et al., An integrated sensing and wireless communications platform for sensing sodium in sweat. Anal. Methods 8, 64-71 (2016). https://doi.org/10.1039/C5AY02254A 